\title{
Graphene Field-Effect Transistor Based High-Performance Wireless Portable Device for HIN1 Detection
}

\author{
Received 11 May, 2021; revised 8 June, 2021; accepted 1 July, 2021
}

\author{
Kyung Ho Kim ${ }^{a, \dagger}$, Jai Eun An ${ }^{a, \dagger}$, Jun-Seob Kim ${ }^{b}$, Joonwon Bae ${ }^{c, *}$, and Oh Seok Kwon ${ }^{a, d, *}$ \\ a Infectious Disease Research Center, Korea Research Institute of Bioscience and Biotechnology (KRIBB), Daejeon \\ 34141, Republic of Korea \\ bDepartment of Nano-Bioengineering, Incheon National University, Incheon 22012, Republic of Korea \\ 'Department of Applied Chemistry, Dongduk Women's University, Seoul 02748, Republic of Korea \\ d Nanobiotechnology and Bioinformatics (Major), University of Science \& Technology (UST), Daejeon 34141, Republic \\ of Korea \\ †Contributed equally to this work as first author.
}

*Corresponding author E-mail: oskwon7799@gmail.com, joonwonbae@gmail.com

\begin{abstract}
In this study, a convenient high-performance portable sensor platform for simple, fast, and efficient detection of H1N1 virus is demonstrated using a graphene-based transistor type architecture. A uniform graphene layer was generated and patterned by conventional methods such as lithography and vapor deposition, subsequently, electrodes were introduced on the patterned graphene layer to obtain transistor type sensor geometry. Then, the graphene surface was functionalized with antibody for H1N1 virus detection and sensor performance test. The transition curve, linearity, and sensitivity ( $10 \mathrm{pfu} / \mathrm{mL}$ ) of the sensor component were measured. In addition, the portable H1N1 diagnosis platform for simple, fast, and convenient virus detection was produced and demonstrated. Consequently, the sensor performance was maintained in the portable sensor platform compared with the graphene-based sensor component. This presented portable H1N1 diagnosis platform showed better performance than the lateral flow assay.
\end{abstract}

Keywords: Graphene, Portable biosensor, Field-effect transistor, H1N1

\section{Introduction}

The respiratory viruses may cause various symptoms and diseases such as fever, cough, bronchitis, and pneumonia. Due to the corona19 virus pandemic, the need for ultrasensitive high-performance sensors to detect potentially prevalent respiratory viruses has increased dramatically worldwide [1]. On the other hand, corona and influenza viruses bring similar symptoms, therefore, it is significant to develop elaborated biosensors to detect and distinguish individual virus for accurate diagnosis at early stage. Even if diverse methodologies and devices to diagnose harmful viruses have been proposed and studied, it is still a challenging task because of the sensitivity and accuracy is less competitive than the molecular diagnosis such as polymerase chain reaction [2]. Lateral flow assay (LFA) is also a good candidate for virus detection, but its performance is still far from that required for practical use $[3,4]$. Therefore, it is necessary to produce a novel biosensor showing high sensitivity and selectivity to a specific virus. Moreover, portal or mobile biosensors are more desirable to minimize infection and to suppress spread at the same time.

To date, our research group has reported extensive research activities regarding demonstration of the graphene-based biosensors detecting diverse biomaterial, such as hormone, biomolecule, virus, and pathogens [5-7]. Therefore, it has been proved that graphene can be used effectively for biosensors with sophisticated structure and geom- etry. Because graphene is patternable and its surface can be modified for functionalization, various sensing media such as receptor, aptamer, and antibody can be incorporated for effective target capture $[8,9]$. In addition, introduction of electrodes with various geometry can lead to a transistor type sensor [10]. By using the transistor structure, sensor performance such as sensitivity, linearly, and selectivity can be elevated remarkably. Also, underlying inherent sensor characteristics such as transition curve can be obtained.

In this work, a convenient high-performance portable sensor platform was produced using graphene-based field effect transistor type sensor module for selective detection of $\mathrm{H} 1 \mathrm{~N} 1$ virus. The sensor platform was built as a 3-layer device, in which each layer conducted designated functions. Graphene was produced by the chemical vapor deposition (CVD) and played a role of conductive substrate for signal transduction. Transistor structure was obtained by conventional patterning of graphene layer and vapor deposition of electrodes. The successful formation of sensor geometry was proved using diverse instrumental analyses such as transmission electron microscopy (TEM) and Raman spectroscopy. The sensing medium, antibody was attached to the graphene surface via a simple functionalization using adhesive molecules. The substantial incorporation of antibody was confirmed by FT-IR spectroscopy and electrical measurement. The performance of graphene-based sensor module was measured by electrical measurement. In addition, the feasibility of portal sensor platform con- 
taining the graphene-based sensor module was also demonstrated using a mobile phone interface. This study can provide really important information for future research regarding high-performance portable virus sensors, which can be used effectively and extensively.

\section{Experimental details}

\subsection{Materials and Instruments}

Copper foil (0.001 in thick) was purchased from Alfa Aesar. Glutaraldehyde (GA) and ammonium persulfate (APS) was purchased from Sigma Aldrich Co., Ltd. 950 PMMA A4 4 \% in anisole, was purchased from Micro Chem Co., Ltd. H1N1 antibody (ab84612) was purchased by Abcam plc. Lateral flow assay (STANDARD Q Influenza A/B) was purchased from Biosensor, INC. High-resolution transmission electron microscopy (HR-TEM) images were obtained with FEI Tecnai instrument to figure out the monolayer graphene through wet transfer process onto copper grid. Raman spectroscopy were measured with a BX41RF-LED instrument (Olympus, $633 \mathrm{~nm}$ laser) to further prove the single layer of the used graphene. FT-IR were obtained with AlphaP model for surface modification.

\subsection{Graphene fabrication}

Single layer graphene was fabricated on the copper foil by CVD method using $\mathrm{H}_{2}, \mathrm{CH}_{4}$, and Ar gases. After, single layer graphene was transferred using wet transfer process, PMMA was removed by hot acetone vapor.

\subsection{Fabrication of H1N1 Ab-conjugated FET}

The electrodes were fabricated through micro electromechanical system process. First, 4 inch wafer with single layer graphene transferred was coated with photoresist followed by baking with respective condition. Photolithography was used for graphene micropatterning followed by the development of the pattern. To micropattern the graphene and to form the FET channel, reactive-ion etching was used. Electrode patterning was performed through photolithography to form source/drain pattern. Metal $(\mathrm{Cr} / \mathrm{Au})$ deposition was demonstrated with E-beam to form source/drain and underwent the lift-off process using acetone. Formation of passivation layer was performed with photolithography followed by $\mathrm{SiO}_{2}$ deposition and lift-off process. After the final process, $4 \mu \mathrm{L} \mathrm{GA}(2 \%)$ was dropped on the graphene channel for $4 \mathrm{~h}$ at $4{ }^{\circ} \mathrm{C}$, then $4 \mu \mathrm{L}$ of H1N1 antibody (Ab, $1 \mu \mathrm{g} / \mathrm{mL}$ ) was treated on the bis(2-aminoethylene)perylene-3,4,9,10tetracarboxyldiimide (PDA)-conjugated graphene for $4 \mathrm{~h}$ at $4{ }^{\circ} \mathrm{C}$.

\subsection{Virus culture}

The influenza H1N1 virus was grown in the chicken eggs for 3 days at $37^{\circ} \mathrm{C}$, cultured virus was collected by using centrifuge $4,000 \mathrm{rpm}$, $10 \mathrm{~min}, 4^{\circ} \mathrm{C}$. The collected $\mathrm{H} 1 \mathrm{~N} 1$ virus was counted through plaque assay, the counted virus was diluted using phosphate buffered saline ( $\mathrm{pH}$ 7.4) solution to $10 \mathrm{pfu} / \mathrm{mL}$.

\section{Results and discussion}

\subsection{Preparation of graphene substrate}

In this study, a graphene layer played several important roles such as substrate, signal transduction, and providing electrical conductivity. The graphene layer is generally advantageous for these purposes owing to its inherent properties such as high conductivity, heat dissipation, facilitated charge transport, and chemical/physical inertness [11-13]. In this experiment, a graphene layer was produced by the chemical vapor deposition method. This method is considered superior to the chemical reduction method [14-17]. A copper substrate was used after strict cleaning and chemical vapor deposition process was operated according to the designated cycle under argon atmosphere as depicted in Fig. 1(a), which was composed of annealing, growth, and cooling step. The gas feed ratios were selected as results of repetitive exper- a)
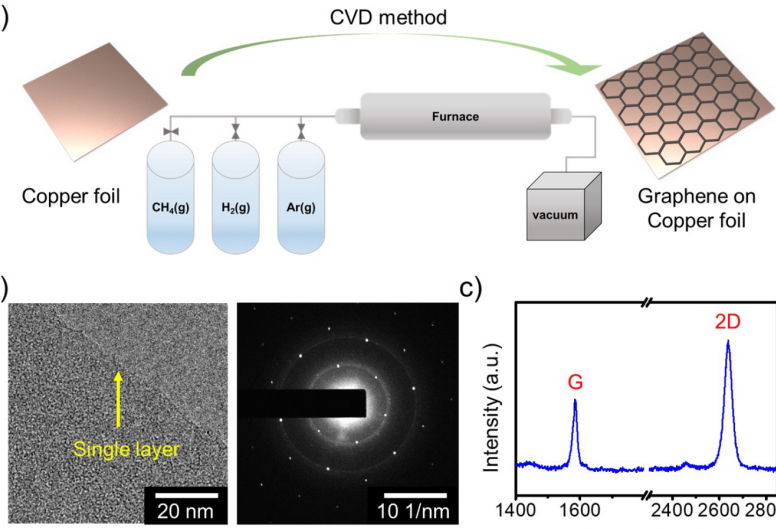

Graphene on Copper foil

b)

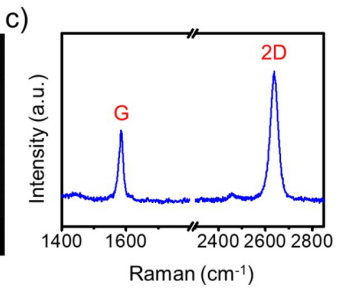

Figure 1. Characterization of graphene. (a) Schematic diagram of CVD graphene fabrication, (b) TEM and selected area electron diffraction images of single layer graphene, and (c) Raman spectrum of single layer graphene.

iments in our group. When the chemical vapor deposition was conducted under the conditions, a single layer graphene was formed as confirmed in our previous study [18]. TEM image of the graphene layer is shown in Fig. 1(b). A uniform and homogeneous layer was observed extensively and there was no trace of regional buckling and dewetting. Electron diffraction image in Fig. 1(b) also shows that the graphene layer possessed a highly crystalline structure, because perfect hexagonal diffraction patterns were obtained. Raman spectrum in Fig. 1(c) also supports that the nature of prepared layer was graphitic as observed at around $1600 \mathrm{~cm}^{-1}$, while a peak associated with an amorphous character also appeared at $2650 \mathrm{~cm}^{-1}$ [19]. It was demonstrated that a graphene layer was effectively produced on the original substrate.

\subsection{Fabrication of a sensor module on the graphene layer}

Figures 2(a) and 2(b) shows the digital images of the prepared sensor and its electrode structure. First, the graphene layer was patterned to an array of ribbons with a high aspect ratio using conventional lithography and etching methods. Subsequently, Au electrodes such as source and drain were introduced via vapor deposition. A gate

a)
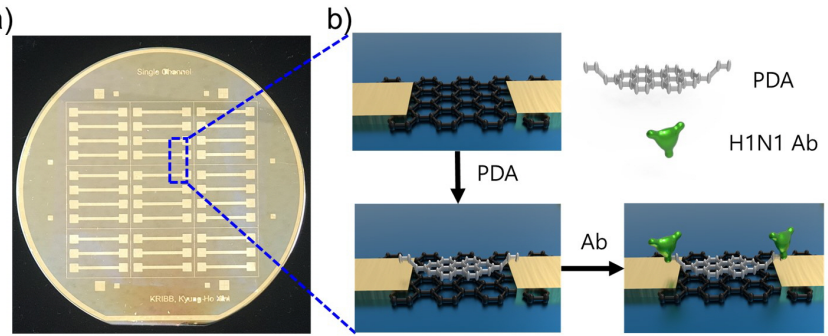

c)

d)
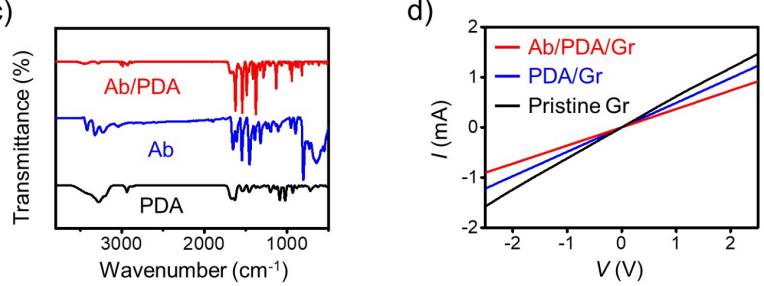

Figure 2. Characterization of Ab conjugated FET. (a) Photography of large-scale electrodes, (b) schematic diagram of the modification of graphene surface, (c) FT-IR spectra depending on the surface modification process, and (d) I-V curves depending on the surface modification process. 
a)

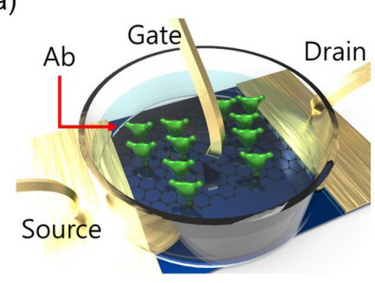

c)

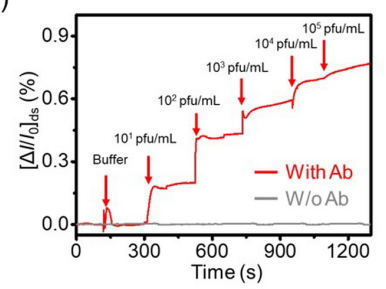

b)

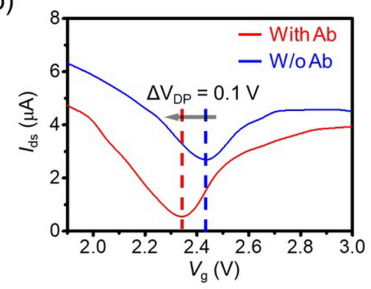

d)

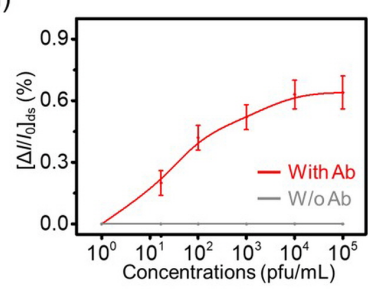

e)

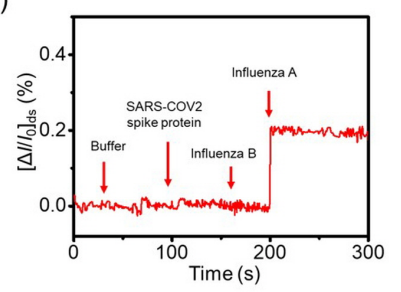

Figure 3. (a) Illustration of liquid-ion gated system, (b) transfer curves before and after Ab immobilization, (c) real-time responses toward various H1N1 concentrations, (d) calibration curve of the H1N1 Ab-conjugated FET, and (e) real-time response for selectivity toward influenza A with the H1N1 Ab-conjugated FET.

electrode could be applied perpendicular to the graphene layer to obtain transistor geometry, which will be described in Fig. 3. Subsequently, the graphene surface was modified with a series of chemicals to incorporate a sensing medium, H1N1 antibody. First, the surface was treated with PDA and GA. Then antibodies were attached to the surface for detection and immobilization of target viruses to the surface. Once this phenomenon occurs spontaneously, an electrical signal must be induced between source and drain. This is because the electrical properties such as resistance and conductance changes after addition of virus to the antibody. This signal can be transported to the controlling system through an electrical circuit. When a gate electrode is used to a transistor system, this signal can be amplified effectively. Therefore, sensor performance can be improved without addition of high concentration analyte.

The successful preparation of the sensor device was analyzed with FT-IR spectroscopy and electrical measurement. The FT-IR spectra in Fig. 2(c) show the characteristic peaks for PDA, antibody, and antibody/PDA layer. Note that the peak locations were totally different. In addition, the contribution of antibody was significantly higher than that of PDA as shown in the spectrum of the antibody/PDA (red color), because the antibody layer was located on top of the PDA layer. The ohmic relation curves in Fig. 2(d) indicates that the introduction of PDA layer increased the resistivity of the system, thus the slope of PDA/graphene (Gr) (blue color) decreased compared with that of pristine Gr. This meant that the PDA layer acted a role of resistance to the graphene layer leading to an increase in resistivity [20]. Because the antibody molecules were also electrical insulating, therefore, this layer also acted as a resistance to the graphene layer. Consequently, the slope of antibody/PDA/Gr (red color) increased compared with other curves. This trend was consistent with the FT-IR spectroscopy result.

a)

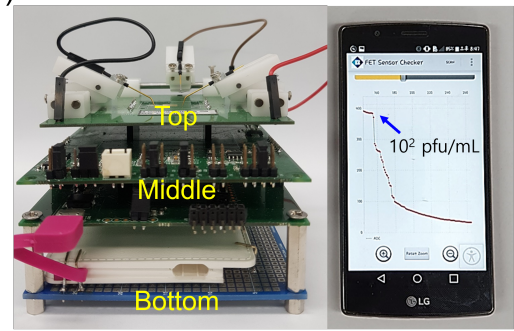

b)

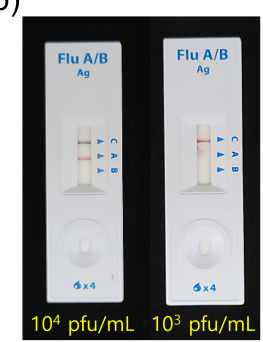

Figure 4. (a) Photography of wireless portable device based on graphene fieldeffect transistor (left) and real-time response of $10^{2} \mathrm{pfu} / \mathrm{mL}$ H1N1 using smart phone (right), and (b) photography of the results toward H1N1 detection for diverse concentrations using LFA kits.

\subsection{Performance of the transistor type sensor device and portable platform}

The transistor type sensor geometry used in this study is described in Fig. 3(a). As mentioned above, use of the gate electrode is desirable for stable signal transduction and amplification. In addition, the auxiliary container is necessary for the introduction of liquid analyte, because H1N1 viruses needs to be added as buffer solution. Even if the effective attachment of antibody to the graphene surface was confirmed by FT-IR and ohmic relation, supplementary transition curve measurement was conducted before and after antibody attachment [Fig. 3(b)]. It was clear that the transition voltage decreased approximately $0.1 \mathrm{~V}$ from 2.43 to $2.33 \mathrm{~V}$. This was associated with the change in the electrical properties the graphene surface due to the presence of antibodies. The sensitivity tests were conducted with varying H1N1 concentration. When the antibody was absent, there was no signal associated with the antibody-virus interactions. On the contrary, the signal increased steadily after addition of H1N1 virus from 10 to $10^{5}$ $\mathrm{pfu} / \mathrm{mL}$. When a blank buffer solution was added, a peak appeared and became extinct rapidly to the original level. However, the baseline rose and maintained after addition of $\mathrm{H} 1 \mathrm{~N} 1$ virus. Conversion of the data in Fig. 3(c) as a function of concentration led to a linearity curve in Fig. 3(d). While there was no variation without antibody, the correlation was remarkable after antibody addition. Selectivity of the sensor to the H1N1 virus was already secured with the selection of antibody. The selectivity of Ab-conjugated FET sensor platform was performed by injection of another interference virus [Fig. 3(e)]. Although there is no significant signal toward interference viruses such as influenza B and SARS-COV2 spike protein, the response obviously showed toward influenza A.

The diagnosis performance of portable sensor platform is demonstrated in Fig. 4. The mobile platform is organized as a three-story stacked structure. The bottom layer contains an auxiliary battery to provide electric power for on-site operations. The middle layer has a printed circuit board (PCB), which controls the overall operation of device and have an interface with mobile phones. The top layer is a three-electrode probe to measure sensitivity and linearity of the graphene-based sensor module. Once a signal was detected by the sensor module, the result data was processed by the PCB board on the second layer and communicated to the mobile phone as a digital value. The mobile phone window indicated that it was possible to detect $10^{2}$ $\mathrm{pfu} / \mathrm{mL}$ virus using the mobile sensor platform. The validity of the graphene-based sensor device was further demonstrated by manufacture of lateral flow assay with the H1N1 antibody. A distinct red line appeared at control sample ("C"), but a line was not observed for "A" at the virus concentration of $10^{3} \mathrm{pfu} / \mathrm{mL}$. On the contrary, a red line was observed both for "C" and " $\mathrm{A}$ ", when the virus concentration was increased to $10^{4} \mathrm{pfu} / \mathrm{mL}$. Those observations indicated that the detection limit of the LFA was $10^{4} \mathrm{pfu} / \mathrm{mL}$ and the sensitivity of the mobile sensor platform was $10^{2}$ times higher than that of LFA device. 


\section{Conclusions}

In this work, a portable diagnosis platform to detect $\mathrm{H} 1 \mathrm{~N} 1$ viruses using a graphene-based ultrasensitive high performance sensor device was manufactured and its performance was also demonstrated compared with lateral flow assay. The graphene layer prepared via chemical vapor deposition played a role of conductive substrate. Introduction of electrodes and surface functionalization of graphene resulted in the formation of transistor type sensor geometry. The sensor device showed a sensitivity of $10 \mathrm{pfu} / \mathrm{mL}$ with a linear region $10 \sim 10^{5} \mathrm{pfu} / \mathrm{mL}$. The selectivity of the sensor device was obtained by a specific antibody. In addition, a portable platform was produced by assembly of external battery, printed circuit board and mobile phone interface, and sensor device. It showed a detection limit of $10^{2} \mathrm{pfu} / \mathrm{mL}$, which was $10^{2}$ times higher than that of lateral flow assay. This work can provide essential information for future research regarding portable virus diagnosis.

\section{Acknowledgements}

This research was supported by the Defense Acquisition Program Administration (ADD-911255201)

\section{References}

[1] J. Sachs et al., Lancet 396, 1102 (2020).

[2] V. M. Corman et al., Eurosurveillance 25, 23 (2020).

[3] X. Zhu et al., Biosens. Bioelectron. 166, 112437 (2020).

[4] L. J. Carter et al., ACS Cent. Sci. 6, 591 (2020).

[5] S. J. Park, S. E. Seo, K. H. Kim, S. H. Lee, J. Kim, S. Ha, H. S. Song,
S. H. Lee, and O. S. Kwon, Biosens. Bioelectron. 174, 112804 (2021).

[6] K. H. Kim, S. H. Lee, S. E. Seo, J. Bae, S. J. Park, and O. S. Kwon, Micromachines 11, 439 (2020).

[7] S. J. Park, O. S. Kwon, S. H. Lee, H. S. Song, T. H. Park, and J. Jang, Nano Lett. 12, 5082 (2012).

[8] N. Nakatsuka et al., Science 362, 319 (2018).

[9] D. K. H. Tsang, T. J. Lieberthal, C. Watts, I. E. Dunlop, S. Ramadan, A. E. D. R. Hernandez, and N. Klein, Sci. Rep. 9, 13946 (2019).

[10] J. Park, H. H. Nguyen, A. Woubit, and M. Kim, Appl. Sci. Converg. Technol. 23, 61 (2014).

[11] F. Schwierz, Nat. Nanotechnol. 5, 487 (2010).

[12] Y. Zhu, S. Murali, W. Cai, X. Li, J. W. Suk, J. R. Potts, and R. S. Ruoff, Adv. Mater. 22, 3906 (2010).

[13] S. H. Lee, K. H. Kim, S. E. Seo, M. I. Kim, S. J. Park, and O. S. Kwon, J. Ind. Eng. Chem. 83, 29 (2020).

[14] O. S. Kwon et al., Nano Lett. 15, 6559 (2015).

[15] O. S. Kwon et al., Adv. Mater. 25, 4177 (2013).

[16] S. Bae, S. K. Lee, and M. Park, Appl. Sci. Converg. Technol. 27, 79 (2018)

[17] H. Ko, J. S. Lee, and S. M. Kim, Appl. Sci. Converg. Technol. 27, 144 (2018).

[18] K.H. Kim et al., Biosens. Bioelectron. 167, 112514 (2020).

[19] B. J. Lee, S. I. Jo, and G. H. Jeong, Appl. Sci. Converg. Technol. 27, 100 (2018).

[20] S. J. Park et al., Sci. Rep. 10, 3772 (2020). 